\title{
Effects of multidisciplinary integrated care on quality of care in residential care facilities for elderly people: a cluster randomized trial
}

\author{
Marijke Boorsma MD, Dinnus H.M. Frijters PhD, Dirk L. Knol PhD, Miel E. Ribbe MD, Giel Nijpels MD, \\ Hein P.J. van Hout PhD
}

See related commentary by Stadnyk and colleagues at www.cmaj.ca/lookup/doi/10.1503/cmaj.110789.

Competing interests: None declared.

This article has been peer reviewed.

Correspondence to: Hein P.J. van Hout, hpj.vanhout@vumc.nl

CMAJ 2011. DOI:10.1503 /cmaj.101498

\section{ABSTRACT}

Background: Sophisticated approaches are needed to improve the quality of care for elderly people living in residential care facilities. We determined the effects of multidisciplinary integrated care on the quality of care and quality of life for elderly people in residential care facilities.

Methods: We performed a cluster randomized controlled trial involving 10 residential care facilities in the Netherlands that included 340 participating residents with physical or cognitive disabilities. Five of the facilities applied multidisciplinary integrated care, and five provided usual care. The intervention, inspired by the disease management model, consisted of a geriatric assessment of functional health every three months. The assessment included use of the Long-term Care Facility version of the Resident Assessment Instrument by trained nurseassistants to guide the design of an individualized care plan; discussion of outcomes and care priorities with the family physician, the resi- dent and his or her family; and monthly multidisciplinary meetings with the nurse-assistant, family physician, psychologist and geriatrician to discuss residents with complex needs. The primary outcome was the sum score of 32 riskadjusted quality-of-care indicators.

Results: Compared with the facilities that provided usual care, the intervention facilities had a significantly higher sum score of the 32 quality-of-care indicators (mean difference $-6.7, p=0.009$; a medium effect size of 0.72 ). They also had significantly higher scores for 11 of the 32 indicators of good care in the areas of communication, delirium, behaviour, continence, pain and use of antipsychotic agents.

Interpretation: Multidisciplinary integrated care resulted in improved quality of care for elderly people in residential care facilities compared with usual care.

Trial registration: www.controlled-trials.com trial register no. ISRCTN11076857.
$\mathrm{T}$ he quality of care provided in residential care facilities is under pressure worldwide. ${ }^{1}$ Facilities are frequently understaffed, and the complexity of care needed by residents increases while expertise of staff does not necessarily keep pace. ., $^{2,3}$ Although most care organizations want to innovate and improve quality of care, many lack expertise or financial resources needed to do so. ${ }^{4,5}$ Family physicians are responsible for medical care in residential care facilities in the Netherlands. However, they do not regard themselves as suited for systematic management of chronic diseases and disabilities associated with frail health. ${ }^{6}$

About $10 \%$ of elderly people aged 75 or older in the Netherlands live in residential care facilities. $^{7.8}$ These facilities were established to offer sheltered living for elderly people who are disabled but still relatively healthy. Because of the growing elderly population, the characteristics of elderly people living in residential care facilities have become more comparable to those of people in nursing homes, who need complex care. Residential care facilities in the Netherlands are comparable to residential care facilities in Canada, are publicly funded and are subject to government inspection and approval. Over $70 \%$ of the residents need professional care, such as assistance with activities of daily living, nursing care (e.g., medication, wound care) and housekeeping. They have multiple chronic diseases and associated disabilities. ${ }^{9-12}$

Effective interventions for chronic illnesses generally rely on a multidisciplinary team ap- 
proach. The elements of this approach include structured geriatric assessment, protocol-based regulation of medications, support for self-reliance and intensive follow-up. The closely related disease management model comprises coordination of care, steering of the care process and patient empowerment. ${ }^{13}$ This model is strongly recommended by Bodenheimer and colleagues to improve the health and quality of life of chronically ill patients. ${ }^{14}$ However, no studies have as yet been undertaken to evaluate the effects of disease management on functional health and quality of care for elderly people in residential care facilities who have physical or cognitive disabilities.
We developed an approach to multidisciplinary integrated care inspired by the disease management model. The objective of our study was to determine the effects of multidisciplinary integrated care on quality of care and quality of life for elderly people in residential care facilities.

\section{Methods}

The study was approved by the ethics committee of the VU University Medical Center in Amsterdam, the Netherlands. All participating residents or their proxies provided written informed consent. Figure 1 shows the flow of participants through the study.

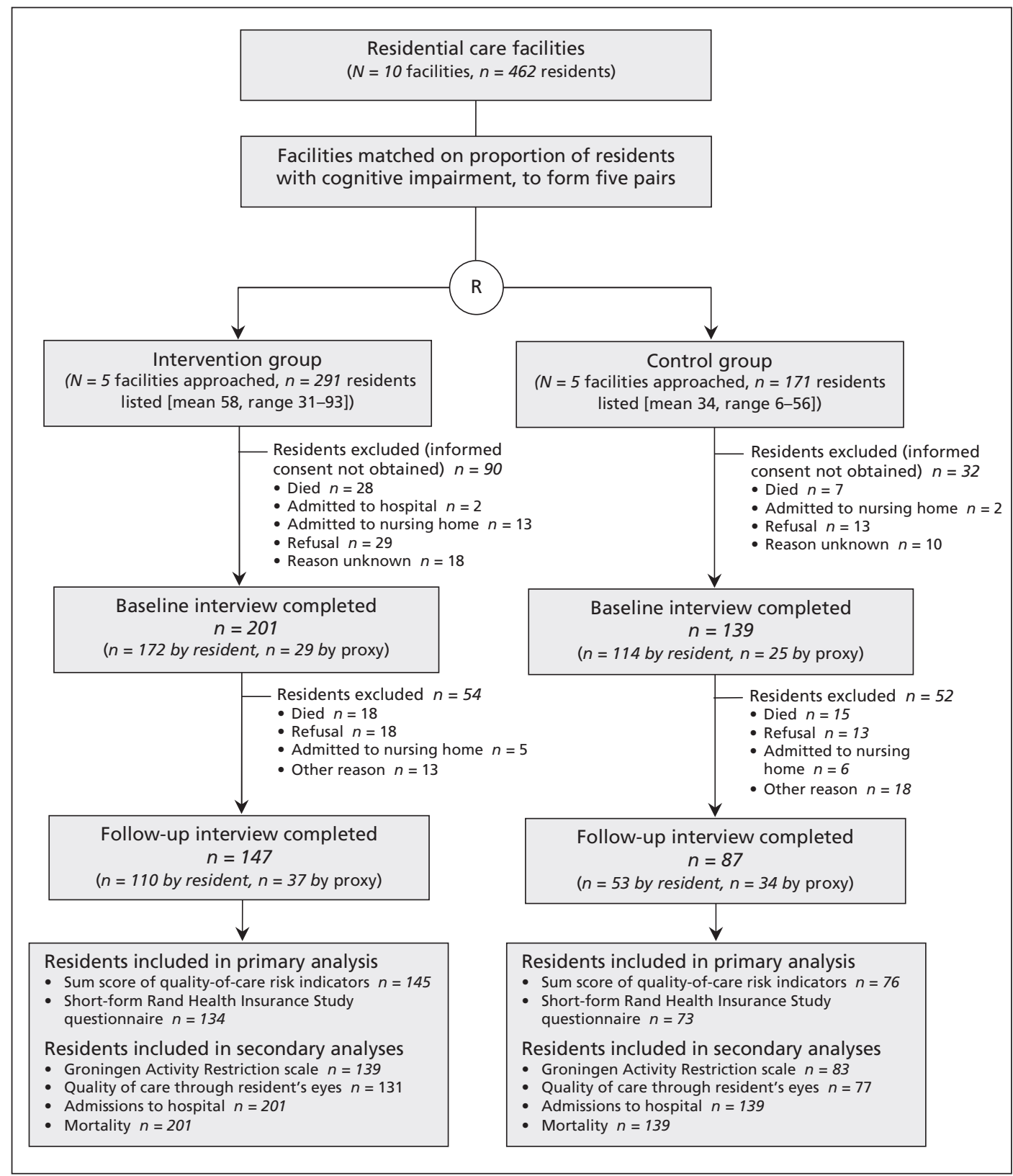

Figure 1: Flow of completed interviews of participants through the trial. Because of missing data, the numbers of residents included in the primary and secondary analyses differed from the numbers interviewed. $\mathbf{R}=$ randomization. 


\section{Study design and participants}

After a pilot study in one residential care facility, a cluster randomized controlled clinical trial was set up involving 10 facilities belonging to the umbrella care organization Omring, a large home care and long-term care provider in the Netherlands. The pilot facility was excluded from the trial. Randomization was carried out at the facility level; five facilities were assigned to the intervention group and the other five to the usual care group.

A total of 462 residents from the 10 facilities were recruited from December 2006 until December 2007. The average number of residents in each facility was 46 , and staff included nurseassistants and a house manager. All residents were listed in a primary care practice whose physician was responsible for their medical care. Residents who were terminally ill (as determined by staff or family physician) were excluded from the study.

Participating residents in each facility were visited by trained, blinded interviewers at baseline and at six months. If the resident was unable to understand the questions, a close family member was identified by staff and asked to act as proxy. The interview consisted of a computerized assessment of functional health, activities of daily living, depression, cognition, satisfaction with care, and use of medications. Proxies completed the interview except for the cognitive assessment, which was replaced by a short form of the Informant Questionnaire on Cognitive Decline in the Elderly. ${ }^{15}$

The duration of the trial was relatively short because of a high risk for dropout owing to the extreme vulnerability of residents and because the umbrella care organization intended to implement the care model in the control facilities as well. A detailed description of the design was reported earlier. ${ }^{16}$

\section{Randomization}

Randomization was carried out on facilities after matching for percentage of cognitively impaired residents, based on the assumption that a high percentage of such residents would affect care-related needs and services. In the matching procedure, the two facilities with the highest percentage of cognitively impaired residents were matched, and so on. Randomization was carried out using the first column from Pocock's random numbers table. ${ }^{17}$

\section{Intervention}

By adapting the principles of disease management, we introduced the concept of multidisciplinary integrated care. This concept focused on identification and monitoring of the functional disabilities caused by chronic diseases. Its three basic elements correspond to those of the disease management model: monitoring of disabilities, coordination of care and empowerment. ${ }^{13}$ The third element is normally applied to patients only. However, we wanted to empower nurse-assistants in relation to monitoring and coordination of care, given that they provided all basic nursing care.

The model of multidisciplinary integrated care used in our study comprised five elements. ${ }^{16}$ First, a geriatric multidimensional assessment of all residents was conducted every three months. The Web-based Long-term Care Facility version 9.0 of the Resident Assessment Instrument was used for this purpose..$^{18}$ The identified problem areas guide the design of an individualized care plan that is intended to improve or maintain functional health status. Second, the care plan was discussed with the resident, the resident's family and the family physician, and adapted to personal wishes. Third, residents with complex care needs were scheduled at least twice a year for a multidisciplinary meeting. Fourth, consultation with a geriatrician or psychologist was optional for the frailest residents with complex health care problems. Fifth, data from the Webbased Resident Assessment Instrument was used to provide an overview every three months of 32 risk-adjusted quality-of-care indicators. These indicators were compared with benchmark values derived from data on all residents of residential facilities in the Netherlands obtained using this instrument. ${ }^{19,20}$ Further details of the model of multidisciplinary integrated care are found in Appendix 1 (available at www.cmaj.ca/lookup /suppl/doi:10.1503/cmaj.101498/-/DC1).

\section{Usual care}

For facilities assigned to usual care, the family physician was responsible for medical care and offered it on request. There was neither coordination nor structured planning of care. Multidisciplinary meetings were mostly not attended by the family physicians.

\section{Outcome measures}

For the purpose of the evaluation, experienced, specially trained, blinded and supervised interviewers independently assessed the residents at baseline and six months later. The interviewers' assessments were supplemented by systematic observations by staff and extraction of data from residents' medical records (e.g., actual medication regimen).

\section{Primary outcome measures}

The first primary outcome was the sum score of the 32 risk-adjusted quality-of-care indicators, 
which were developed by Morris ${ }^{21}$ and showed good validity and reliability. Appendix 2 (available at www.cmaj.ca/cgi/content/full/cmaj $.101498 / \mathrm{DC} 1)$ shows the definitions of the quality-of-care indicators, including their numerator and denominators. The quality-ofcare indicators were originally based on observations recorded in the Long-term Care Facility assessment form. We incorporated the itemized observations needed to calculate these indicators in the assessments performed by our independently trained interviewers. Interrater reliability of the quality-of-care indicators between interviewers and nurse-assistants in the intervention facilities was satisfactory (mean intracluster correlation single measure 0.74 ). The sum score of the quality-of-care indicators was determined by the number of indicators per resident divided by the number of indicators applied to a resident. Indicators were calculated using mixed linear hierarchical models.

Four of the 32 quality-of-care indicators (behaviour problem, bladder or bowel incontinence, pressure ulcer and use of antipsychotics) applied both to the group of residents as a whole and to high- and low-risk groups. We therefore calculated two sum scores, one for all 32 indicators and one for the 24 whole-group indicators after exclusion of the 8 that were broken down to apply to high- and low-risk groups. Of these 24 whole-group indicators, on average 21 (standard deviation [SD] 6.6) applied to the residents. Of all 32 indicators, on average 22 (SD 6.1) applied to the residents. The relatively lower number of applicable indicators among all 32 indicators is due to the inclusion of the breakdown indicators, which applied to a maximum of $50 \%$ of the residents. The Cronbach's $\alpha$ of the sum score of the 24 whole-group indicators was 0.62 .

For the second primary outcome, healthrelated quality of life was measured using a short-form 12-item version of the Rand Health Insurance Study questionnaire..$^{22}$ Its properties were satisfactory when used by proxies, which was important in our study because of the percentage of cognitively impaired residents $(58.2 \%){ }^{23}$ We also calculated the number of quality-adjusted life-years using the algorithm of Hatoum and colleagues. ${ }^{24}$

\section{Secondary outcome measures}

The secondary outcome measures comprised the 32 individual risk-adjusted quality-of-care indicators (described in Appendix 2); activities of daily living, as measured by the Groningen Activity Restriction Scale designed for elderly respondents and validated by Kempen and coauthors ${ }^{25}$ quality of care from the residents' per- spective, as measured by a short (16-item) version of the QUOTE-Elderly instrument (Quality of Care from the Perspective of the Elderly), ${ }^{26}$ hospital admissions recorded at the (single) local hospital, which covered more than $95 \%$ of all admissions $;{ }^{16}$ and mortality, as recorded by the interviewers or staff and cross-checked by the administration of the municipality.

\section{Process outcomes}

Process outcomes comprised the percentage of residents with completed assessments; the number of multidisciplinary meetings held, based on minutes of the meetings; the numbers of agreedon medical, nursing and social actions, based on content analysis of care plans; and opinions of participating professionals regarding the intervention protocol, as obtained by interviews with staff and family physicians.

\section{Statistical analysis}

\section{Sample-size calculations}

Sample-size calculations were based on the expected effects of the intervention on quality of care and functional health using an $\alpha$ level of 0.05 , a power of $80 \%$, a dropout rate of $15 \%$ and an anticipated intracluster correlation of 0.05 , based on Adams and colleagues, ${ }^{27}$ across the residential facilities. To detect a fair benefit (i.e., Cohen's $d$ effect size of 0.5 ), we estimated that the sample should include at least 82 residents in each of the two study groups. ${ }^{28}$

\section{Effect analyses}

Effect analyses were performed according to both intention-to-treat and per-protocol principles. We accepted that the protocol was adhered to when the first two (obligatory) steps of the intervention were performed. We compared differences in the outcome measures over six months between the intervention and control groups using multivariable techniques. We applied mixed models for the continuous outcomes, using an unstructured covariance matrix for the longitudinal data. For the dichotomous outcomes, we applied generalized estimating equations using a logit link and an exchangeable working correlation.

In all effect analyses, we adjusted for baseline imbalance (e.g., by age, sex, cognitive impairment, depression, disability and interview by proxy). The analyses were done with multilevel specification if these variables showed significantly higher log-likelihood estimates. Because of our cluster randomized design (with randomization at the facility level), results of multilevel and "simple" analyses were compared for all outcomes. If higher-order clustering effects were 
present, outcomes of the multilevel analyses were presented; if clustering was negligible, results of "simple" analyses were presented.

The intracluster coefficient across facilities was estimated by exchangeable working correlation for all individual (dichotomous) quality-ofcare indicators. In all outcomes with pre-post

\begin{tabular}{|c|c|c|}
\hline Characteristic & $\begin{array}{l}\text { Intervention } \\
\text { group }\end{array}$ & $\begin{array}{l}\text { Control } \\
\text { group }\end{array}$ \\
\hline Facilities & $N=5$ & $N=5$ \\
\hline No. of participating residents, mean (SD) & $39.8(17.7)$ & $27.2(18.0)$ \\
\hline $\begin{array}{l}\text { No. of personnel, full-time equivalent } \\
\text { per resident, mean (SD) }\end{array}$ & $0.33(0.04)$ & $0.44(0.10)$ \\
\hline Residents & $n=201$ & $n=139$ \\
\hline Age, yr, mean (SD) & $85.8(6.2)$ & $85.5(8.0)$ \\
\hline Sex, male, no. (\%) & $48(23.9)$ & $36(25.9)$ \\
\hline Marital status, no. (\%) & $n=191$ & $n=135$ \\
\hline Married & $42(22.0)$ & $27(20.0)$ \\
\hline Widowed & $130(68.1)$ & $93(68.9)$ \\
\hline Never married & $19(9.9)$ & $15(11.1)$ \\
\hline Education, no. (\%) & $n=190$ & $n=132$ \\
\hline Primary school or less & $112(58.9)$ & $79(59.8)$ \\
\hline Lower technical vocational training & $45(23.7)$ & $26(19.7)$ \\
\hline Average and higher vocational training & $34(17.9)$ & $30(22.7)$ \\
\hline Clinical characteristics, no. (\%) & $n=199$ & $n=136$ \\
\hline Asthma or COPD & $29(14.6)$ & $13(9.6)$ \\
\hline Myocardial infarction & $61(30.7)$ & $30(22.1)$ \\
\hline Cerebrovascular accident & $33(16.6)$ & $29(21.3)$ \\
\hline Diabetes & $39(19.6)$ & $27(19.9)$ \\
\hline Arthritis & $73(36.7)$ & $47(34.6)$ \\
\hline Cancer & $12(6.0)$ & $11(8.1)$ \\
\hline Hypertension & $35(17.6)$ & $32(23.5)$ \\
\hline Chronic somatic diseases, no., mean (SD) & $1.54(1.25)$ & $1.49(1.17)$ \\
\hline Cognitive impairment* & $101(50.8)$ & $90(66.2)$ \\
\hline Clinical depressiont & $10 \quad(5.0)$ & $16(11.8)$ \\
\hline Body mass index, mean (SD) & $\begin{array}{c}n=164 \\
26.2(4.98)\end{array}$ & $\begin{array}{c}n=116 \\
26.3(4.67)\end{array}$ \\
\hline Falls experienced in past 30 days, no. (\%) & $n=190$ & $n=134$ \\
\hline One & $23(12.1)$ & $18(13.4)$ \\
\hline Two or more & $8(4.2)$ & $10(7.5)$ \\
\hline Total & $31(16.3)$ & $28(20.9)$ \\
\hline Medications, no., mean (SD) & $7.6(3.4)$ & $8.0(3.6)$ \\
\hline 4-m walking time, sec, median (IQR) & $4.0(1.0-7.75)$ & $5.0(1.0-8.0)$ \\
\hline Not able to do walking test & $\begin{array}{l}n=172 \\
76(44.2)\end{array}$ & $\begin{array}{c}n=114 \\
59(51.8)\end{array}$ \\
\hline \multicolumn{3}{|c|}{$\begin{array}{l}\text { Note: } \mathrm{COPD}=\text { chronic obstructive pulmonary disease, IQR = interquartile range, SD = standard } \\
\text { deviation. } \\
\text { *Score of }<5 \text { on the Memory Impairment Screen, or } \geq 3.6 \text { on the short form of the Informant } \\
\text { Questionnaire on Cognitive Deterioration. } \\
\text { tDiagnosed by family physician or specialist. }\end{array}$} \\
\hline
\end{tabular}

measurements, the effect of interest was the group $\times$ time (pre-post) interaction effect. A $p$ value of 0.05 was considered to be significant.

\section{Process analyses}

We evaluated the extent to which the intervention program was performed according to proto$\mathrm{col}$, the nature of the recommendations of the multidisciplinary meeting and the judgments of family physicians and staff about the intervention program.

\section{Results}

\section{Sample and facility characteristics}

Baseline characteristics of the residents and facilities are described in Table 1. There was a small imbalance between the intervention group and the usual care group in the numbers of residents with cognitive impairment and in the numbers with clinical depression. The trial ended up imbalanced because one control facility did not accept new entries as a result of an upcoming move to another location, and because a second control facility was in the middle of moving to a new building and could therefore recruit few residents for the study. Analyses without these facilities did not change the results.

\section{Primary outcomes}

Compared with residential care facilities that provided usual care, the intervention facilities had a significantly higher sum score of the 32 risk-adjusted quality-of-care indicators (mean difference -6.7 (95\% confidence interval [CI] -8.69 to $-4.71, p=0.009$; Cohen $d$ effect size 0.72) (Table 2). Self-reported quality of life did not differ between residents of control and intervention facilities (Table 3 ).

\section{Secondary outcomes}

The intervention facilities had higher scores than the control facilities for 30 of the 32 riskadjusted indicators of quality of care; the scores for 11 of these 30 indicators had increased significantly (Table 2). In the intention-to-treat analyses, no differences in disability or quality of care as seen through residents' eyes were found between the two groups of facilities (Table 3). In the per-protocol analysis, residents in the intervention facilities tended to be more positive about the quality of care over time than residents in the usual care facilities (difference 1.8, $p=0.072$ ). The per-protocol analyses showed that fewer residents died in the intervention group than in the control group (intervention $10 / 112$, control 25/139; odds ratio $2.15,95 \%$ CI $1.06-4.38 ; p=0.035)$. 
Table 2: Risk-adjusted indicators of quality of care for elderly people in intervention and control residential care facilities during the six-month study period

\begin{tabular}{|c|c|c|c|c|}
\hline \multirow[b]{2}{*}{ Indicator } & \multirow[b]{2}{*}{$\begin{array}{l}\text { Intracluster } \\
\text { coefficient }\end{array}$} & \multicolumn{2}{|c|}{ Group; \% of residents } & \multirow[b]{2}{*}{$\begin{array}{l}\text { Adjusted OR* } \\
\quad(95 \% \mathrm{Cl})\end{array}$} \\
\hline & & $\begin{array}{l}\text { Intervention } \\
\text { facilities }\end{array}$ & $\begin{array}{l}\text { Control } \\
\text { facilities }\end{array}$ & \\
\hline $\begin{array}{l}\text { Worsening of activities } \\
\text { of daily living }\end{array}$ & 0.02 & 14.7 & 48.5 & $0.17(0.07-0.40)$ \\
\hline Worsening of locomotion & $<0.001$ & 16.1 & 7.1 & $2.85(0.91-8.96)$ \\
\hline Increase in no. of falls & -0.03 & 17.6 & 9.6 & $2.00(0.69-5.64)$ \\
\hline Decline in cognitive ability & 0.03 & 18.4 & 24.1 & $0.84(0.51-1.38)$ \\
\hline Decline in communication & 0.01 & 17.5 & 46.9 & $0.25(0.13-0.49)$ \\
\hline Delirium, new or persistent & 0.06 & 28.2 & 56.3 & $0.27(0.10-0.69)$ \\
\hline Behavioural problem & 0.01 & 8.7 & 26.5 & $0.27(0.10-0.74)$ \\
\hline High risk & 0.04 & 15.4 & 45.5 & $0.24(0.07-0.89)$ \\
\hline Low risk & 0.04 & 2.0 & 8.6 & $0.20(0.03-1.34)$ \\
\hline Little or no social activity & - & 0.0 & 0.0 & - \\
\hline New in-dwelling catheter & -0.03 & 1.0 & 4.6 & $0.04(0.006-0.29)$ \\
\hline In-dwelling catheter & 0.01 & 2.0 & 0.0 & - \\
\hline Bladder or bowel incontinence & -0.04 & 33.3 & 46.2 & $0.78(0.66-0.91)$ \\
\hline High risk & -0.06 & 62.5 & 71.1 & $0.79(0.28-2.28)$ \\
\hline Low risk & -0.06 & 19.4 & 34.1 & $0.52(0.40-0.67)$ \\
\hline Decline in bowel continence & -0.03 & 6.9 & 23.4 & $0.25(0.15-0.45)$ \\
\hline Decline in bladder continence & -0.02 & 14.6 & 45.9 & $0.17(0.07-0.38)$ \\
\hline Urinary tract infection & 0.01 & 1.9 & 4.5 & $0.79(0.25-2.49)$ \\
\hline Use of feeding tube & $<0.001$ & 0.0 & 1.5 & - \\
\hline Low body mass index & $<0.001$ & 1.9 & 0.0 & - \\
\hline Weight loss & 0.001 & 3.9 & 4.6 & $0.67(0.09-5.00)$ \\
\hline Inadequate pain management & -0.01 & 21.4 & 13.2 & $1.81(0.91-3.57)$ \\
\hline Worsening of pain & -0.03 & 12.9 & 40.9 & $0.20(0.07-0.52)$ \\
\hline Presence of pressure ulcers & -0.01 & 4.9 & 7.5 & $0.63(0.21-1.91)$ \\
\hline High risk & -0.17 & 16.0 & 19.0 & $0.80(0.18-3.44)$ \\
\hline Low risk & 0.02 & 1.3 & 2.1 & $0.51(0.06-4.04)$ \\
\hline Worsening of pressure ulcers & 0.03 & 1.9 & 5.2 & $0.57(0.31-1.05)$ \\
\hline Burns, skin tears or cuts & 0.02 & 8.7 & 4.5 & $1.9 \quad(0.91-4.14)$ \\
\hline Use of physical restraints & - & 0.0 & 1.5 & - \\
\hline Use of antipsychotic agents & 0.01 & 3.8 & 11.0 & $0.25(0.08-0.78)$ \\
\hline High risk & 17.00 & 25.0 & 25.0 & $0.89(0.03-36.1)$ \\
\hline Low risk & 0.01 & 2.1 & 10.7 & $0.15(0.03-0.66)$ \\
\hline Sum score & & & & Mean difference $(95 \% \mathrm{Cl})$ \\
\hline $\begin{array}{l}\text { For all } 32 \text { quality-of-care indicators, } \\
\text { range } 0 \%-100 \% \text { (SD)†‡ }\end{array}$ & 0.01 & $11.5(9.0)$ & $18.2(9.4)$ & $-6.7(-8.69$ to -4.71$)$ ๆ \\
\hline $\begin{array}{l}\text { For } 24 \text { whole-group quality-of-care } \\
\text { indicators, range } 0 \%-100 \% \text { (SD) }+\S\end{array}$ & 0.05 & $9.1(9.4)$ & $15.7(11.2)$ & $-6.6(-8.81$ to -4.39$)$ ** \\
\hline \multicolumn{5}{|c|}{$\begin{array}{l}\text { Note: } \mathrm{Cl}=\text { confidence interval, } \mathrm{OR}=\text { odds ratio, } \mathrm{SD}=\text { standard deviation. } \\
\text { *Calculated by generalized linear models. ORs and sum scores for quality-of-care risk indicators were adjusted for sex, age, interview by proxy, depression and } \\
\text { cognitive impairment at baseline, accounting for within-facility clustering. } \\
\text { tThe sum score was determined by the number of quality-of-care indicators per resident divided by the number of quality-of-care indicators applied to a resident } \\
\text { Indicators were calculated using mixed linear hierarchical models. } \\
\text { fOf the } 32 \text { quality-of-care indicators, a mean of } 23.1 \text { applied to residents in intervention facilities and } 20.1 \text { applied to residents in control facilities. } \\
\text { SThe } 24 \text { whole-group quality-of-care indicators excludes the } 8 \text { indicators broken down to apply to high- and low-risk groups. Of the } 24 \text { whole-group indicators, } \\
\text { a mean of } 21.7 \text { applied to residents in intervention facilities and } 19.5 \text { applied to residents in control facilities. } \\
\text { If } p=0.009 \text {. } \\
* * p=0.021 .\end{array}$} \\
\hline
\end{tabular}




\section{Process of care}

The first step of the protocol - assessment with the Long-term Care Facility version of the Resident Assessment Instrument - was completed for $55.2 \%$ of the residents in the intervention facilities. This proportion was less than we had aimed for and was partly a result of implementation delay.

Forty multidisciplinary meetings were held in the intervention facilities during the study period, and the outcomes of assessment of 93 residents included in the study were discussed (Table 4 ). The primary care physician was present at $90 \%$ of the multidisciplinary meetings, and the geriatrician at $75 \%$. By contrast, only $25 \%$ of the multidisciplinary meetings in the control facilities were visited by the primary care physician. The number of recommended actions per resident was 3.67 in the intervention facility meetings and 2.26 in the control facility meetings.

The expertise of staff was felt by $52.9 \%$ of staff and $54.5 \%$ of the family physicians to have increased after introduction of the care model. The same percentage of staff and $63.6 \%$ of family physicians felt that they had more knowledge about residents' health. In addition, $58.8 \%$ of staff and $81.8 \%$ of the family physicians felt that their cooperation had improved. About 55\% of the family physicians considered quality of care to have improved; $73 \%$ acknowledged the need for a new care model.

\section{Ancillary analyses}

We did not find effect modification of the outcomes by age, sex or baseline disability.

\section{Interpretation}

Compared with usual care, our model of multidisciplinary integrated care resulted in substantially higher quality of care for elderly people in residential care facilities. Functional ability, number of hospital admissions and health-related quality of life remained comparable between the two groups. According to the per-protocol analyses, mortality was lower in the intervention facilities and residents in the intervention facilities were more positive about their quality of care. Owing to the short intervention period (six months), the full protocol was applied to less than half of the residents in the intervention facilities. The training and empowerment of nurse-assistants, which was completed for all intervention facilities, together with monitoring using the geriatric assessment instrument, were likely to be the most important ingredients for improvement of the quality of care.

Earlier studies have reflected elements of our intervention. For example, positive health effects on residents have been reported as a result of interdisciplinary geriatric primary care in American facilities. ${ }^{29}$ Integrated and home-based geriatric care management was reported to improve quality of care and reduce use of acute care ser-

\begin{tabular}{|c|c|c|c|c|c|}
\hline Outcome measure & $\begin{array}{l}\text { Intracluster } \\
\text { coefficient }\end{array}$ & $\begin{array}{l}\text { Intervention } \\
\text { group }\end{array}$ & $\begin{array}{l}\text { Control } \\
\text { group }\end{array}$ & Effect statistic & $p$ value \\
\hline Continuous (range) & & \multicolumn{2}{|c|}{ Mean (SD) } & Group $\times$ time & \\
\hline $\begin{array}{l}\text { Short } 12 \text {-item version of the Rand Health } \\
\text { Insurance Study questionnaire }(0-100)\end{array}$ & 0.02 & & & 1.02 & 0.35 \\
\hline Baseline & & $43.34(5.96)$ & $42.33 \quad(6.86)$ & & \\
\hline $6 \mathrm{mo}$ & & $42.31(6.04)$ & $42.56 \quad(6.35)$ & & \\
\hline Quality-adjusted life-years (0-1) & 0.05 & & & & \\
\hline $6 \mathrm{mo}$ & & $0.28(0.11)$ & $0.27 \quad(0.12)$ & 0.87 & \\
\hline Groningen Activity Restriction scale (18-72) & 0.13 & & & -1.40 & 0.16 \\
\hline Baseline & & $43.70(13.73)$ & $39.90(13.91)$ & & \\
\hline $6 \mathrm{mo}$ & & $42.41(13.37)$ & $39.06(13.70)$ & & \\
\hline Quality of care through residents' eyes (16-64) & 0.07 & & & 1.56 & 0.12 \\
\hline Baseline & & $57.41(5.46)$ & $58.01 \quad(6.69)$ & & \\
\hline $6 \mathrm{mo}$ & & $56.32(6.47)$ & $56.10 \quad(6.64)$ & & \\
\hline Dichotomous & & & $(\%)$ & OR $(95 \% \mathrm{Cl})$ & \\
\hline Mortality & -0.02 & 28/201 (13.9) & 25/139(18.0) & $1.09(0.87-1.38)$ & 0.44 \\
\hline Admissions to hospital $\geq 1$ & -0.02 & $22 / 142(15.5)$ & $12 / 85(14.1)$ & $1.32(0.94-1.87)$ & 0.11 \\
\hline
\end{tabular}


vices in a high-risk group of low-income elderly people living at home. ${ }^{30,31}$ Use of the Home Care version of the Resident Assessment Instrument in primary care health centres in Hong Kong resulted in improvement in 2 of 13 functional outcomes. $^{32}$ Modest positive effects on wellbeing and on deterioration of functional skills were found in a multidisciplinary program in vulnerable older people living at home. ${ }^{33}$

\section{Limitations}

Our study was limited by the fact that our population was frail and comprised a high percentage of cognitively impaired residents. As a result, a portion of the data was collected from interviews with proxies. The judgments of proxies may have differed from the residents' judgments. Therefore, we adjusted for proxy interview and cognitive status in our analyses. The cluster randomization produced an imbalance between the intervention and control facilties in the number of participating residents and in some of the functional characteristics of the residents at baseline. Although we adjusted for the imbalance in functional characteristics, imbalance in the number of participating residents may have led to underpowered results.

Variation across the intervention facilities in the application of the complete protocol (3\%-66\%) was another limitation. This variation can be explained by financial and administrative issues during the study period. The financial obligations for residential care facilities resulting from a new national funding system for residential care of elderly people caused uncertainty about job continuation, high turnover of managers and new priorities at the facilities in our study. Despite this limitation, the improvement in quality of care at the facilities in our study was substantial.

\section{Conclusion}

Our model of multidisciplinary integrated care resulted in improved quality of care for elderly people in residential care facilities compared with usual care. The results of this study are applicable to elderly people in such settings as residential care facilities and nursing homes, and even elderly people living in the community. In primary care settings, it may be beneficial to have a model to monitor elderly people and those with chronic diseases, to prevent functional decline and admission to hospital for acute care. It is also important to have an instrument that not only delivers output on the patient level but also on the management level, to facilitate monitoring of quality of care by managers in a sector of health care that is under enormous societal pressure to improve its performance.
Table 4: Characteristics and outcomes of multidisciplinary meetings held during the six-month study period

\begin{tabular}{|lcc|}
\hline Characteristic or outcome & $36(90)$ & $7(25)$ \\
\hline $\begin{array}{l}\text { Meetings at which primary care } \\
\text { physician was present, no. (\%) } \\
\text { facility meetings } \\
n=40\end{array}$ & $\begin{array}{c}\text { Control facility } \\
\text { meetings } \\
n=28\end{array}$ \\
\hline $\begin{array}{l}\text { Meetings at which geriatrician was } \\
\text { present, no. (\%) }\end{array}$ & $30(75)$ & $21(75)$ \\
\hline $\begin{array}{l}\text { Meetings at which psychologist was } \\
\text { present, no. (\%) }\end{array}$ & $21(53)$ & $16(57)$ \\
\hline $\begin{array}{l}\text { No. of residents discussed, total } \\
\text { (per meeting) }\end{array}$ & $93(2.1)$ & $68(2.4)$ \\
\hline $\begin{array}{l}\text { No. of actions on care plan, total } \\
\text { (per resident) }\end{array}$ & $92(0.99)$ & $60(0.88)$ \\
\hline Medical & $124(1.33)$ & $27(0.40)$ \\
\hline Nurse care & $32(0.34)$ & $12(0.18)$ \\
\hline Referral to medical specialist & $53(0.57)$ & $37(0.41)$ \\
\hline Paramedical referral & $40(0.43)$ & $18(0.25)$ \\
\hline Medication change & $341(3.67)$ & $154(2.26)$ \\
\hline All & & \\
\hline
\end{tabular}

\section{References}

1. Bos JT, Frijters DH, Wagner C, et al. Variations in quality of home care between sites across Europe, as measured by home care quality indicators. Aging Clin Exp Res 2007;19:323-9.

2. Broese van Groenou MI. Unequal chances for reaching "a good old age." Socio-economic health differences among older adults from a life course perspective [article in Dutch]. Tijdschr Gerontol Geriatr 2003;34:196-207.

3. Spector WD, Cohen JW, Pesis-Katz I. Home care before and after the Balanced Budget Act of 1997: shifts in financing and services. Gerontologist 2004;44:39-47.

4. Challis D, Hughes J. Residential and nursing home care issues of balance and quality of care. Int J Geriatr Psychiatry 2003;18:201-4.

5. Challis D, Stewart K, Donnelly M, et al. Care management for older people: Does integration make a difference? J Interprof Care 2006;20:335-48.

6. Challis D, Hughes J. Frail old people at the margins of care: some recent research findings. Br J Psychiatry 2002;180:126-30.

7. Pot AM, Portrait F, Visser G, et al. Utilization of acute and longterm care in the last year of life: comparison with survivors in a population-based study. BMC Health Serv Res 2009;9:139.

8. Tomassini C, Glaser K, Wolf DA, et al. Living arrangements among older people: an overview of trends in Europe and the USA. Popul Trends 2004; Spring:24-34.

9. Geerlings SW, Pot AM TJ, Deeg DJG. Predicting transitions in the use of informal and professional care by older adults. Ageing Soc 2005;25:111-30.

10. Simonsick EM, Kasper JD, Phillips CL. Physical disability and social interaction: factors associated with low social contact and home confinement in disabled older women (the Women's Health and Aging Study). J Gerontol B Psychol Sci Soc Sci 1998; 53:S209-17.

11. Sprangers MA, de Regt EB, Andries F, et al. Which chronic conditions are associated with better or poorer quality of life? J Clin Epidemiol 2000;53:895-907.

12. Strawbridge WJ, Sheme SJ, Balfour JL, et al. Antecedents of frailty over three decades in an older cohort. J Gerontol B Psychol Sci Soc Sci 1998;53:9-16.

13. Bijl D. Effectiveness of disease management programmes for recognition, diagnosis and treatment of depression in primary care. Eur J Gen Pract 2004;10:6-12.

14. Bodenheimer $\mathrm{T}$, Wagner $\mathrm{EH}$, Grumbach K. Improving primary care for patients with chronic illness: the chronic care model, Part 2. JAMA 2002;288:1909-14.

15. Jorm AF. A short form of the Informant Questionnaire on Cognitive Decline in the Elderly (IQCODE): development and cross-validation. Psychol Med 1994;24:145-53. 
16. Boorsma M, van Hout HP, Frijters DH, et al. The cost-effectiveness of a new disease management model for frail elderly living in homes for the elderly: design of a cluster randomized controlled clinical trial. BMC Health Serv Res 2008;8:143.

17. Pocock S. Clinical trials: a practical approach. New York (NY): Wiley and Sons; 1983.

18. Hirdes JP, Fries BE, Morris JN, et al. Integrated health information systems based on the RAI/MDS series of instruments. Healthc Manage Forum 1999;12:30-40.

19. Frijters D, Gerritsen D, Steverink N, et al. Care quality: reliability and usefulness of observation data in bench marking nursing homes and homes for the aged in the Netherlands. Tijdschr Gerontol Geriatr 2003;34:21-9.

20. Zimmerman DR. Improving nursing home quality of care through outcomes data: the MDS quality indicators. Int J Geriatr Psychiatry 2003;18:250-7.

21. Morris J. Validation of long-term and post-acute care quality indicators, final report. Cambridge (MA): Abt Associates Inc.; Rosalind (MA): HRCA Research and Training Institute; Providence (RI): Brown University Center for Gerontology and Health Care Research; 2003.

22. Brook RH, Ware JE Jr, Davies-Avery A, et al. Overview of adult health status measures fielded in Rand's Health Insurance Study. Med Care 1979;17(Suppl 7):1-131.

23. Brazier JE, Harper R. Jones NM, et al. Validating the SF-36 health survey questionnaire: new outcome measure for primary care. BMJ 1992;305:160-4

24. Hatoum HT, Brazier JE, Akhras KS. Comparison of the HUI3 with the SF-36 preference based SF-6D in a clinical trial setting. Value Health 2004;7:602-9.

25. Kempen GI, Miedema I, Ormel J, et al. The assessment of disability with the Groningen Activity Restriction Scale. Conceptual framework and psychometric properties. Soc Sci Med 1996;43:1601-10.

26. Sixma HJ. Quality of care from the perspective of elderly people: the QUOTE-Elderly Instrument. Age Ageing 2000;29:173-8.

27. Adams G. Patterns of intra-cluster correlation from primary care research to inform study design and analysis. J Clin Epidemiol 2004:57:785-94.

28. Cohen J. Statistical power analysis for the behavioural sciences. New York (NY): Academic Press; 1977.

29. Burns R, Nichols LO, Graney MJ, et al. Impact of continued geriatric outpatient management on health outcomes of older veterans. Arch Intern Med 1995;155:1313-8.

30. Counsell SR, Callahan CM, Clark DO, et al. Geriatric care management for low-income seniors: a randomized controlled trial. JAMA 2007;298:2623-33.

31. Counsell SR, Callahan CM, Tu W, et al. Cost analysis of the Geriatric Resources for Assessment and Care of Elders care management intervention. J Am Geriatr Soc 2009;57:1420-6.

32. Chi I, Chou KL, Kwan CW, et al. Use of the Minimum Data Set - Home Care: a cluster randomized controlled trial among the Chinese older adults. Aging Ment Health 2006;10:33-9.

33. Melis RJ, van Eijken MI, Borm GF, et al. The design of the Dutch EASYcare study: a randomised controlled trial on the effectiveness of a problem-based community intervention model for frail elderly people [NCT00105378]. BMC Health Serv Res 2005;5:65.

Affiliations: From the Departments of General Practice (Boorsma, Nijpels, van Hout), Nursing Home Practice (Frijters, Ribbe), and Epidemiology and Biostatistics (Knol), EMGO Institute for Health and Care Research, VU University Medical Center, Amsterdam, the Netherlands

Contributors: Marijke Boorsma, Hein van Hout and Giel Nijpels were involved in the conception and design of the study. Marijke Boorsma and Dinnus Frijters were involved in the acquisition of the data. Marijke Boorsma, Hein van Hout, Dirk Knol and Miel Ribbe were involved in the analysis and interpretation of the data. Marijke Boorsma and Hein van Hout were involved in the drafting of the article. All of the authors were involved in the critical revision of the manuscript for important intellectual content and approved the final version submitted for publication.

Funding: This study was funded by the Netherlands Organization for Health Research and Development (ZonMw grant no. 945-05-030). The funding organization had no role in the design and conduct of the study; the collection, management, analysis and interpretation of the data, or the preparation, review and approval of the manuscript. 